\title{
Unique Clinicopathology of Proximal Gastric Carcinoma: A Critical Review
}

Qin Huang

Department of Pathology and Laboratory Medicine, Veterans Affairs Boston Healthcare

System and Harvard Medical School, West Roxbury, Mass., USA

\section{Key Words}

Carcinoma - Chinese · Gastric cancer · Gastric cardia · Proximal stomach

\begin{abstract}
Background: Gastric cancer is a heterogeneous disease with respect to its molecular and histopathological features. Proximal gastric carcinoma (PGC) and distal gastric carcinoma (DGC) are two distinct clinical entities, suggesting the existence of different pathogenic mechanisms. PGC arises in a narrow region of the proximal stomach below the gastroesophageal junction. It accounts for around half of gastric cancers in men, with an increasing incidence worldwide and a predominance in elderly males. Summary: At present, the pathogenic mechanisms involved in the onset of PGC remain unknown. This mini-review presents the most recent findings on the pathology and natural history of this widespread and frequently fatal cancer. Key Message: PGC has unique clinicopathological characteristics distinct from esophageal adenocarcinoma and DGC. Practical Implications: Patients with a high risk for PGC, such as elderly obese men, should undergo upper endoscopy for early detection and appropriate endoscopic therapy in the early stages of disease. Once it has progressed, the cancer is more easily spread, although the current staging systems are not perfectly adapted to the disease. PGC should be staged and treated as a gastric cancer. A separate staging system and genomic studies on this cancer are urgently needed for optimal patient management and appropriate disease prevention.

(C) 2014 S. Karger AG, Basel
\end{abstract}

\section{Introduction}

Despite considerable advances in medical sciences in recent years, gastric cancer remains the world 4th most common cancer with dismal prognosis, ranking the second leading cause of cancer death worldwide [1]. Compared to a low incidence of gastric cancer in Western 
countries, Eastern Asian countries are among the high-risk regions [1]. In addition to substantial geographic variations among different countries, gastric cancer is also well known for heterogeneity in clinicopathology, survival and molecular pathobiology between proximal gastric carcinoma (PGC) and distal gastric carcinoma (DGC), suggesting the existence of different pathogenesis mechanisms for these two distinct groups of gastric cancer. PGC, also known as gastric cardia carcinoma, is common in Eastern Asians and arises in a narrow region of the proximal stomach below the gastroesophageal junction (GEJ). At present, the pathogenesis mechanism for PGC remains largely unknown. The results of previous investigations, primarily carried out in Western countries, show a remarkable similarity between PGC and distal esophageal adenocarcinoma (EAC) in epidemiology [2, 3], clinicopathology [4] and molecular pathobiology [5]. As such, the 7th edition of the American Joint Committee on Cancer (AJCC) staging manual has classified PGC as a part of the EAC spectrum [6]. In that staging manual, the entire section on PGC has been removed from the chapter of gastric cancer to that of esophageal cancer, requiring staging PGC as EAC, based on the assumption that PGC is derived from the short-segment Barrett's esophagus. This dramatic shift in paradigm has a considerable impact upon not only the pathological diagnosis of PGC, but also the management and prevention of PGC patients worldwide. In this mini-review, we present the most recent evidence in pathology on this common, frequently fatal cancer.

\section{Incidence and Risk Factors}

Over the past 60 years, a rapidly rising incidence in both PGC and EAC has been observed in the United States, European countries and Australia, where gastric cancer is uncommon [1], but PGC accounts for about half of gastric cancers in men [1, 7], showing epidemiology characteristics, such as an increasing trend, elderly male predominance and Caucasian race, similar to EAC but different from DGC. Therefore, many Western investigators advocate that both PGC and EAC belong to the same disease entity and should be staged and treated alike. It must be pointed out that almost all previous reports include PGC tumors that are frequently large, over $5 \mathrm{~cm}$ in maximum dimension and often destroy the landmarks of the GEJ $[3,8]$, which makes a confident exclusion of EAC very difficult, let alone an accurate pathological diagnosis of PGC. Because of uncertainty on the correct pathological classification between PGC and EAC, the precision of previous Western epidemiology study data on PGC is doubtful. This concern is echoed in a recent epidemiology study carried out in a well-defined Swedish population, in which the authors reported a misclassification of PGC as EAC in a substantial number of cases, leading to a falsely increased incidence of EAC in that population [9]. Such variations in pathological classification of gastric cancer by location may be present among different countries and also over various time periods, which has complicated interpretation of geographic and secular trends in PGC epidemiology, especially in cancer registries of many countries in which gastric cancer location, i.e. PGC or DGC, is not specified in over $20 \%$ of gastric cancer cases [1].

Unlike in Western countries, EAC in Eastern Asian countries, such as Japan, Korea and China, is rare and exhibits a low and stable incidence over past decades [1,10-12]. In contrast, PGC is very common in those Asian countries [1]. Analysis of the data from the national hospital network in Japan for the period between 1980 and 1994 reveals a decreasing trend in EAC from $2.4 \%$ in 1980 to 1.4\% in 1994 [10]. On the other hand, the data from the National Cancer Center Hospital over the period 1962-1997 show a significantly increased trend in the incidence of PGC. For all early gastric cancers, the proportion of true PGC increased from $0.4 \%$ in 1962 to $4.5 \%$ in 1997, an 11-fold increase [10]. The findings in Japanese PGC patients mirror those in Chinese patients treated at the Nanjing Drum Tower Hospital in China. Under 
guidance of the most recently established histological diagnostic criteria on GEJ [11], Chinese pathologists investigated all distal esophageal carcinoma resection cases over the period 2004-2010 and found EAC in only 1\% of all cases [12]. Furthermore, EAC is not only rare but also stays low in frequency, with no evident changes over the 8-year study period. Most recently, they investigated small PGC with a size $\leq 2 \mathrm{~cm}$ (for an accurate diagnosis of PGC and elimination of misclassification of PGC as EAC) over the time period 2004-2011 and reported a significantly rising trend from $16 \%$ in 2004 to $45 \%$ in 2011 [13]. The data suggest a growing trend in incidence of PGC in contrast to a low and stable occurrence of EAC in both Japan and China in recent years, which is drastically different from that reported in Western patient populations $[1-3,7]$.

Several epidemiology studies carried out mainly in Western countries have demonstrated several risk factors for PGC, such as Caucasian race [14], male gender [14], obesity $[15,16]$, tobacco-alcohol abuse [15], higher socioeconomic status [17] and low fruit and vegetables intake [18]. Intriguingly, Helicobacter pylori infection has been found to be inversely related to EAC and PGC in those countries [19]. However, in Eastern Asian countries, H. pylori remains the major risk factor for PGC. In a large-scale, prospective, case-control epidemiology study in a high-risk region for PGC in China, Kamangar et al. [20] reported a strong association of PGC with $H$. pylori infection, which is in agreement with the results of similar studies in Japan [21] and recent studies in Nanjing, China [13, 22]. In Western countries, while gastroesophageal reflux disease (GERD) has been shown to be a significant risk factor for EAC and GEJ adenocarcinoma, it is not a risk factor for PGC [19]. These findings are comparable to those reported in Chinese patients. Recently, investigators from the Nanjing Drum Tower Hospital in China systematically studied 105 consecutive patients with early PGC for possible risk factors [22]. Although $H$. pylori gastritis was found in a substantial number of PGC cases, the extent of this infection was significantly lower than that in DGCs, suggesting that other risk factors are also at play. The authors further found an older age (average 63.9 years, range 41-84) and a slightly higher male/female ratio of 2.6 in PGC patients, compared to younger age and a lower male/female ratio of 2.1 in DGCs $(n=327)$. Cox multivariate regression analysis revealed several independent risk factors for PGC, such as elderly age ( $>50$ years) (OR 11.9, p < 0.001), overweight (OR 8.2, p < 0.05), obesity (OR 8.0, p < 0.05), history of exposure to industrial toxins (OR 2.6, p < 0.001) and GERD (OR 1.9, p < 0.05) [22]. In contrast, hiatal hernia, tobacco-alcohol abuse, use of non-steroids, low fruit and vegetable intake, hypertension, diabetes, columnar-lined esophagus, intestinal metaplasia and atrophy were not significant risk factors for PGC development [22]. These results parallel those described in Korean patients [23] and are similar to the data described in a recent meta-analysis on the risk of obesity in PGC [24]. In fact, recent epidemiology studies have demonstrated a low occurrence of GERD, hiatal hernia and Barrett's esophagus among the Chinese population [25]. These factors do not play an important role in PGC development in that population.

In summary, although an increased incidence in PGC has been observed globally, the epidemiology evidence for classification of PGC as EAC is insufficient, especially in Eastern Asian patients, because of geographic variations and inaccurate pathological diagnosis of PGC for large tumors among different countries and over various time periods, leading to a frequent misclassification of PGC as EAC. The well-recognized risk factors for PGC in Westerners include Caucasian race, elderly males, obesity, GERD, tobacco-alcohol abuse and high socioeconomic status. By comparison, in Eastern Asian countries EAC is rare but PGC is common. The risk factors of PGC include $H$. pylori infection, elderly males and obesity; the evidence for tobacco-alcohol abuse, hiatal hernia and low fruit-vegetable intake as risk factors for PGC is inadequate or absent. 


\section{Histopathology}

By definition, PGC arises in the cardiac mucosa of the proximal stomach below the GEJ. It should be noted that the gastric cardia is a narrow region with frequent variations among different ethnic populations. Although absent in a substantial proportion of adult Americans [26], the cardiac mucosa has been identified in neonates, children and adults of almost all ethnic populations investigated, and is present in the vast majority of Chinese and Japanese adult subjects [27]. Despite being claimed as a metaplastic lesion due to GERD [4, 26], the cardiac mucosa is still considered as a congenital structure in the proximal stomach with important physiologic function [27]. Malignant progression of the cardiac mucosa forms the basis of PGC.

Endoscopically, early PGC tumors are elevated and protruded [28, 29]. As the disease progresses, the tumor becomes ulcerated with raised borders but remains unifocal in most cases [29]. Once PGC becomes large, straddles and destroys the histological landmark of the GEJ, it is almost impossible to differentiate PGC from EAC $[1,3,8,11]$. In fact, in almost all previous publications on the pathology of PGC, the tumors are large (average size $>5 \mathrm{~cm}$ in maximum dimension), ulcerated and deeply infiltrative [4, 8, 30, 31]. Therefore, it may be almost impossible to be certain whether those large tumors arose in the distal esophagus or in the proximal gastric cardia. It should be emphasized that the differentiation of PGC from EAC is certainly not more of academic interest than of practice value because of the existence of considerable differences in treatment options and prevention strategies of these tumors among different ethnic patient populations.

Because of the lack of accurate pathological information on true PGC in the literature, pathologists in Nanjing, China investigated small gastric cancer $\leq 2 \mathrm{~cm}$ and located within $3 \mathrm{~cm}$ below the GEJ with the most recently established histological criteria of GEJ [11, 13]. In that study, they systematically analyzed 111 consecutive surgical resection specimens for histopathological characteristics of small PGCs and compared these features with those of small DGCs $(\mathrm{n}=202)$ in a homogeneous Chinese patient population. They found a number of unique features of PGC: (1) PGC patients were significantly older with an average age of 63 years (compared to 59.2 years for DGC patients, $\mathrm{p}<0.05$ ), with a significantly higher male/female ratio of 3.1. No PGC patients were younger than 40 years. (2) Endoscopically, PGCs were predominantly unifocal, clustered along the lesser curvature and significantly more common in the protruded form $(\mathrm{p}<0.05)$. (3) PGCs were better differentiated and showed a much wider morphologic spectrum from the predominant adenocarcinoma type (70\%) to uncommon histology varieties of adenosquamous mucinous, medullary and neuroendocrine carcinomas as well as pancreatic acinar-like adenocarcinoma [13, 32]. Poorly cohesive carcinoma, including signet ring cell carcinoma, was significantly less frequent $(\mathrm{p}<0.0001)$. (4) PGCs were also significantly more deeply invasive $(\mathrm{p}<0.05)$ but associated with significantly less frequent lymph node metastasis ( $\mathrm{p}<0.05)$. (5) In the adjacent non-neoplastic gastric mucosa, H. pylori gastritis was common and gastritis cystica profunda was significantly more frequent in PGCs (30\%) than in DGCs (4\%, p < 0.0001); moreover, pancreatic metaplasia was found only with PGCs $(24 \%, \mathrm{p}<0.0001)$. These results in the pathology of PGCs are distinctly different from those of EACs [4, 8, 30] and of DGCs [13]. For instance, EACs, unlike PGCs, are almost exclusively of the adenocarcinoma type; gastritis cystica profunda has not been described in the literature, and pancreatic metaplasia is infrequent, as reported previously $[4,8,30]$.

In summary, the pathological data derived primarily from the investigation of small PGCs in a relatively large sample size in Chinese patients demonstrate unique clinicopathological characteristics and provide, for the first time, detailed pathological information supporting the proposal of classification of PGC as a distinct subtype of gastric, not EAC (at least in Chinese patients) or DGC, as suggested previously by others [33, 34]. 


\section{Natural History, Outcomes and Staging}

PGC is a slow-growing cancer with a natural history of at least 5 years in most cases. In one case report from Japan of an 89-year-old man, the initial endoscopic appearance of PGC was flat, mildly depressed (IIC), as an early gastric cancer [29]. The tumor subsequently progressed to be ulcerated (III) 4 years later with elevated borders and deep submucosal invasion over an 8-year follow-up period [29]. Endoscopic studies in China with long-term follow-ups in patients who, for a variety of reasons, refused surgical resection or chemoradiation therapy showed an estimated medial survival time of 73 months and malignant progression from carcinoma in situ to an advanced stage within about 4-5 years [35]. The 5and 10-year natural survival rates were reported to be 76.5 and 23.5\%, respectively [36]. These survival rates are much better than those reported in Western EAC patients after surgical resection $[1,3,30]$.

The 5-year survival rate in PGC patients after surgical resection was reported to be $70 \%$ in Japanese patients and worse in cases with tumor invasion into the distal esophagus [37, 38]. These results are similar to those in Korean patients [33], but better than those in Chinese patients [30,39]. In a comparison study between PGC in Chinese patients and EAC in American patients [39], although the 3-year survival rate was better in PGCs (54\%) than in EACs (43\%), the 5 -year survival rate was comparable between the two ethnic groups (26 vs. 28\%). Intriguingly, the 5-year stage-by-stage survival comparison for patients at stage III demonstrated a significantly better survival rate in PGC than in EAC patients by multivariate analysis (IIIA: $71 \%$ in PGCs vs. $17 \%$ in EACs; IIIB: 27 vs. $0 \%$; IIIC: 38 vs. $0 \%$; $=0.024, p=0.004$ and $p=$ 0.003 , respectively). These data are almost identical to those described in a recent metaanalysis based on the Surveillance, Epidemiology, and End Results (SEER) database with 1,666 carcinomas (1,474 EACs, 192 PGCs). Whitson et al. [3] reported no significant differences in overall and cancer-specific survival between the EAC and PGC groups. However, this SEER database consisted primarily of American Caucasian patients (73\%) and only 8\% were Asian patients; importantly, the overall survival curve for patients at stage III was conspicuously much better in the PGC than in the EAC group. In early PGCs in Chinese patients [37], the overall median survival after surgery was 48 months. On average, the survival was 45.8 months (range 12-93), smaller than that in the DGC group (51.4 months), but the difference between the two groups was not statistically significant in that study [37]. Regardless, the aforementioned survival results indicate a distinct survival feature in PGC patients, who show a better survival rate than EAC patients at stage III, but a worse overall survival than DGC patients, as also experienced by Japanese researchers [38].

PGC arises in a narrow region below the GEJ and easily crosses the GEJ into the distal esophagus when the tumor becomes large. Once in the esophagus, PGC is required to be staged as an esophageal cancer according to the AJCC staging rule. The validity and effectiveness of this ruling has been seriously challenged, first by pathologists from Nanjing in China [39], and then by Europeans [34] and Koreans [33]. In 142 consecutive Chinese PGC resection tumors that crossed the GEJ and were staged with the rules for EAC, the Kaplan-Meier survival curves for the patients staged at pIIIA showed erroneously better survival than those staged at pIA and pIIB. Moreover, multiple survival curves were crossed for cases staged at pIIB and pIIIB, indicating the existence of inter- and intra-group heterogeneity in survival. Notably, even with the staging rule for gastric cancer, PGC patient survival curves were not distinctive and exhibited incorrectly better survival prediction for cases staged at pIB and pIIB than those at pIA and pIIA. Surprisingly, patients staged at $\mathrm{pN} 3 \mathrm{~B}$ showed an even worse 5-year survival rate than those staged at pM1 and pIV [39]. The results demonstrate a poor discriminatory capability of the AJCC staging rule for PGC patients, who cannot be effectively stratified for prognosis by the current AJCC staging system [33, 34, 39]. Taken together with similar study results from European [34], 
Korean [33] and most recently Canadian patients [40], the PGC tumor staging data from Chinese patients suggest an urgent need for a separate cancer staging system for PGCs.

In summary, an accumulating body of evidence suggests that PGC is a unique, slowgrowing, heterogeneous malignancy that cannot be effectively staged and stratified for prediction of prognosis after surgical resection. Given a worldwide rising incidence of this frequently fatal cancer, a separate cancer staging system is urgently needed for pathological diagnosis, patient management and disease prevention strategies.

\section{Molecular Pathogenesis Mechanism}

Compared to DGC, the pathogenesis mechanism of PGC is poorly understood, involving genetic susceptibility, genetic polymorphisms and environmental factors, including diet among others. Although the pathological investigation of small PGC in a recent study reveals the absence of patients younger than 40 years [13], implying a weak association with a hereditary genetic defect component, several epidemiology studies in China have reported an association of PGC with a positive family history of esophageal squamous cell or gastric carcinoma, in either parents or siblings, with a $40-80 \%$ increased risk [41]. Genetic polymorphism in the interleukin-8 gene may help explain the high susceptibility of elderly men to suffer from PGC, but not esophageal squamous cell carcinomas [42], especially in Asians, but neither in Caucasians nor in Mexicans [43].

With comparative genomic hybridization, Japanese researchers reported significantly more chromosomal gains and losses in 35 PGCs than in 67 DGCs [44]. Interestingly, the most common chromosomal deletion in PGC tumors is on chromosome 18q, in which the Smad gene, a tumor suppressor gene, is located. This gene plays a key role in the transforming growth factor $\beta$ signaling pathway [45]. By immunohistochemistry, the HER2 and SIRT1 genes are also more commonly expressed in PGCs than in DGCs [46, 47]. Moreover, a recent gene expression array study demonstrated several differences in signal transduction pathways between PGC and DGC, compared to the adjacent normal mucosa [40, 48]. Very recently, Canadian researchers used next-generation sequencing technique to study genomic profiles of PGCs and reported in an abstract form the presence of multiple mutation differences, compared to DGCs [40].

In summary, a growing body of evidence derived from the most recent molecular studies suggests a preliminary, but discrete, genomic profile for PGC, which is distinctly different from that for EAC or DGC, suggesting divergent pathogenesis mechanisms for these different cancers.

\section{Conclusions}

At present, mounting evidence in epidemiology, endoscopy, clinicopathology and genomics displays a set of unique clinicopathological characteristics in PGCs, which are distinctly different from those in EACs or in DGCs. In pathology, PGC is a slow-growing, but deeply invasive, heterogeneous malignancy that cannot be effectively stratified for patient postoperative prognosis by the current cancer staging rules. Taken together with distinctive genetic profiles and unique clinicopathological features, PGC should be classified as a distinct subtype of gastric cancer. Further genomic investigations on PGC pathogenetic mechanisms and establishment of a separate cancer staging system for PGC are urgently needed for better patient management and disease prevention, given the worldwide rising incidence of this frequently fatal cancer. 


\section{References}

1 de Martel C, Forman D, Plummer M: Gastric cancer: epidemiology and risk factors. Gastroenterol Clin North Am 2013;42:219-240.

-2 Crew KD, Neugut AI: Epidemiology of gastric cancer. World J Gastroenterol 2006;12:354-362.

-3 Whitson BA, Groth SS, Li Z, Kratzke RA, Maddaus MA: Survival of patients with distal esophageal and gastric cardia tumors: a population-based analysis of gastroesophageal junction carcinomas. J Thorac Cardiovasc Surg 2010;139:43-48.

$\checkmark 4$ Chandrasoma P, Wickramasinghe K, Ma Y, DeMeester T: Adenocarcinomas of the distal oesophagus and 'gastric cardia' are predominantly esophageal carcinomas. Am J Surg Pathol 2007;31:569-575.

5 Wijnhoven BP, Siersema PD, Hop WC, van Dekken H, Tilanus HW: Adenocarcinomas of the distal oesophagus and gastric cardia are one clinical entity. Rotterdam Esophageal Tumour Study Group. Br J Surg 1999;86: 529-535.

6 American Joint Committee on Cancer: Chapter 10: Esophagus and esophagogastric junction; in: AJCC Cancer Staging Manual, ed 7. New York, Springer, 2009, pp 129-144.

7 Crew KD, Neugut AI: Epidemiology of upper gastrointestinal malignancy. Semin Oncol 2004;31:450-464.

-8 Smith RR, Hamilton SR, Boitnott JK, Rogers EL: The spectrum of carcinoma arising in Barrett's esophagus. A clinicopathologic study of 26 patients. Am J Surg Pathol 1984;8:563-573.

-9 Ekström AM, Signorello LB, Hansson LE, Bergström R, Lindgren A, Nyrén O: Evaluating gastric cancer misclassification: a potential explanation for the rise in cardia cancer incidence. J Natl Cancer Inst 1999;91:786-790.

10 Blaser MJ, Saito D: Trends in reported adenocarcinomas of the oesophagus and gastric cardia in Japan. Eur J Gastroenterol Hepatol 2002;14:107-113.

11 Huang Q: Definition of the esophagogastric injunction: a critical mini review. Arch Pathol Lab Med 2011;135: 384-389.

12 Huang Q, Shi J, Sun Q, Fan X, Feng A, Wu H, Zhou Q, Yu C, Mashimo H, Lauwers GY: Distal esophageal carcinomas in Chinese patients vary widely in histopathology, but adenocarcinomas remain rare. Hum Pathol 2012;43: 2138-2148.

13 Shi J, Sun Q, Xu BY, Yu HP, Zhang YF, Zou XP, Huang L, Gold JS, Mashimo H, Yu CG, Huang Q: Changing trends in the proportions of small $(\leq 2 \mathrm{~cm})$ proximal and non-proximal gastric carcinomas treated at a high-volume tertiary medical center in China. J Dig Dis DOI: 10.1111/1751-2980.12151.

14 El-Serag HB, Mason AC, Petersen N, Key CR: Epidemiological differences between adenocarcinoma of the oesophagus and adenocarcinoma of the gastric cardia in the USA. Gut 2002;50:368-372.

-15 Vaughan TL, Davis S, Kristal A, Thomas DB: Obesity, alcohol, and tobacco as risk factors for cancers of the esophagus and gastric cardia: adenocarcinoma versus squamous cell carcinoma. Cancer Epidemiol Biomarkers Prev 1995;4:85-92.

16 Turati F, Tramacere I, LaVecchia C, Negri E: A meta-analysis of body mass index and esophageal and gastric cardia adenocarcinoma. Ann Oncol 2013;24:609-617.

17 Nagel G, Linseisen J, Boshuizen HC, Pera G, Del Giudice G, Westert GP, et al: Socioeconomic position and the risk of gastric and oesophageal cancer in the European Prospective Investigation into Cancer and Nutrition (EPIC-EURGAST). Int J Epidemiol 2007;36:66-76.

-18 Lunet N, Valbuena C, Vieira AL, Lopes C, David L, et al: Fruit and vegetable consumption and gastric cancer by location and histological type: case-control and meta-analysis. Eur J Cancer Prev 2007;16:312-327.

19 Chow WH, Blaser MJ, Blot WJ, Gammon MD, Vaughan TL, et al: An inverse relation between cagA+ strains of Helicobacter pylori infection and risk of esophageal and gastric cardia adenocarcinoma. Cancer Res 1998;58: 588-590.

20 Kamangar F, Qiao YL, Blaser MJ, Sun XD, Katki H, Fan JH, et al: Helicobacter pylori and esophageal and gastric cancers in a prospective study in China. Br J Cancer 2007;96:172-176.

21 Egi Y, Ito M, Tanaka S, et al: Role of Helicobacter pylori infection and chronic inflammation in gastric cancer in the cardia. Jpn J Clin Oncol 2007;37:365-369.

22 Fang C, Sun Q, Shi J, Yu HP, Yu CC, Zhang YF, Zou XP, Huang Q: Clinicopathological risk factors for lymph node metastasis in early gastric carcinoma diagnosed with the WHO criteria in 342 Chinese patients. Gastroenterology 2014;146(5 suppl 1):S-569.

23 Kim JY, Lee HS, Kim N, Shin CM, Lee SH, Park YS, et al: Prevalence and clinicopathologic characteristics of gastric cardia cancer in South Korea. Helicobacter 2012;17:358-368.

24 Chen Y, Liu L, Wang X, Wang J, Yan Z, Cheng J, Gong G, Li G: Body mass index and risk of gastric cancer: a metaanalysis of a population with more than ten million from 24 prospective studies. Cancer Epidemiol Biomarkers Prev 2013;22:1395-1408.

25 Huang Q, Fang DC, Yu CG, Zhang J, Chen MH: Barrett's esophagus-related diseases remain uncommon in China. J Dig Dis 2011;12:420-427.

26 Chandrasoma PT, Der R, Ma Y, Dalton P, Taira M: Histology of the gastroesophageal junction: an autopsy study. Am J Surg Pathol 2000;24:402-409.

27 Huang Q: Controversies of cardiac glands in the proximal stomach: a critical review. J Gastroenterol Hepatol 2011;26:450-455.

28 Mori M, Kitagawa S, Iida M, Sakurai T, Enjoji M, et al: Early carcinoma of the gastric cardia. A clinicopathologic study of 21 cases. Cancer 1987;59:1758-1766. 
Fujisaki J, Nakajima T, Hirasawa T, Yamamoto Y, Ishiyama A, Tsuchida T, et al: Natural history of gastric cancer - a case followed up for eight years: early to advanced gastric cancer. Clin J Gastroenterol 2012;5:351-354.

-30 Huang Q, Fan XS, Agoston AT, Feng AN, Yu HP, Lauwers GY, Zhang LH, Odze RD: Comparison of gastroesophageal junction carcinomas in Chinese versus American patients. Histopathology 2011;59:188-197.

-31 MacDonald WC: Clinical and pathologic features of adenocarcinoma of the gastric cardia. Cancer 1972;29: 724-732.

-32 Huang Q, Gold JS, Shi J, Fan XS, Wu HY, Feng AN, Zhou Q: Pancreatic acinar-like adenocarcinoma of the proximal stomach involving the esophagus. Hum Pathol 2012;43:911-920.

-33 Suh YS, Han DS, Kong SH, Lee HJ, Kim YT, et al: Should adenocarcinoma of the esophagogastric junction be classified as esophageal cancer? A comparative analysis according to the seventh AJCC TNM classification. Ann Surg 2012;255:908-915.

-34 Gertler R, Stein HJ, Loos M, Langer R, Friess H, Feith M: How to classify adenocarcinomas of the esophagogastric junction: as esophageal or gastric cancer? Am J Surg Pathol 2011;35:1512-1522.

-35 Guanrei Y, Songliang Q, He H, Guizen F: Natural history of early esophageal squamous carcinoma and early adenocarcinoma of the gastric cardia in the People's Republic of China. Endoscopy 1988;20:95-98.

-36 Wang GQ, Wei WQ, Zhang JH: Natural progression of early stage adenocarcinoma of gastric cardia: a report of seventeen cases. Ai Zheng 2007;26:1153-1156.

37 Shi J, Sun Q, Yu HP, Zhang YF, Fang C, Yu CC, Zou XP, Mashimo H, Huang Q: Unique clinicopathologic features of early proximal gastric cancer diagnosed with WHO criteria: implications for endoscopic resection. Gastroenterology 2014;146(5 suppl 1):S-515.

-38 Tajima Y, Nakanishi Y, Yoshino T, Kokawa A, Kusano M, et al: Clinicopathological study of early adenocarcinoma of the gastric cardia: comparison with early adenocarcinoma of the distal stomach and esophagus. Oncology 2001;61:1-9.

39 Huang Q, Shi J, Feng AN, Fan XS, Zhang LH, Mashimo H, Cohen D, Lauwers GY: Gastric cardiac carcinomas involving the esophagus are more adequately staged as gastric cancers by the 7 th edition of the American Joint Commission on Cancer Staging System. Mod Pathol 2011;24:138-146.

40 Li-Chang HH, Schaeffer DF, Ng Y, Lum A, Kong E, Kalloger S, et al: Targeted deep sequencing reveals both mutational differences and similarities between gastric cardia and non-cardia adenocarcinomas. Mod Pathol 2014; 27(suppl 2):191A.

41 Li JY, Ershow AG, Chen ZJ, Wacholder S, Li GY, Guo W, et al: A case-control study of cancer of the esophagus and gastric cardia in Linxian. Int J Cancer 1989;43:755-761.

-42 Wang J, Pan HF, Hu YT, Zhu Y, He Q: Polymorphism of IL-8 in 251 allele and gastric cancer susceptibility: a meta-analysis. Dig Dis Sci 2010;55:1818-1823.

43 Savage SA, Abnet CC, Mark SD, Qiao YL, Dong ZW, Dawsey SM, et al: Variants of the IL8 and IL8RB genes and risk for gastric cardia adenocarcinoma and esophageal squamous cell carcinoma. Cancer Epidemiol Biomarkers Prev 2004;13:2251-2257.

-44 Kimura Y, Noguchi T, Kawahara K, Kashima K, Daa T, Yokoyama S: Genetic alterations in 102 primary gastric cancers by comparative genomic hybridization: gain of $20 \mathrm{q}$ and loss of $18 \mathrm{q}$ are associated with tumor progression. Mod Pathol 2004;17:1328-1337.

-45 Nam KT, O’Neal R, Lee YS, Lee YC, Coffey RJ, Goldenring JR: Gastric tumor development in Smad3-deficient mice initiates from forestomach/glandular transition zone along the lesser curvature. Lab Invest 2012;92: 883-895.

46 Fan XS, Chen JY, Li CF, Zhang YF, Meng FQ, Wu HY, et al: Differences of HER2 over-expression between proximal and distal gastric cancers in the Chinese population. World J Gastroenterol 2013;19:3316-3323.

47 Feng AN, Fan XS, Huang Q, Wu HY, Zhang LH: Expression of SIRT1 in gastric cardiac cancer and its clinicopathologic significance. Int J Surg Pathol 2011;19:743-750.

-48 Shah MA, Khanin R, Tang L, Janjigian YY, Klimstra DS, Gerdes H, Kelsen DP: Molecular classification of gastric cancer: a new paradigm. Clin Cancer Res 2011;17:2693-2701. 